\title{
Nanoscale
}

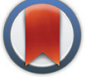

CrossMark

\&lick for updates

Cite this: Nanoscale, 2015, 7, 2900

Received 16th November 2014,

Accepted 13th January 2015

DOI: $10.1039 / c 4 n r 06782 \mathrm{~g}$

www.rsc.org/nanoscale

\section{Laser-ablated titania nanoparticles for aqueous processed hybrid solar cells $\uparrow$}

\author{
V. Körstgens, ${ }^{a}$ S. Pröller, ${ }^{\text {a,b }}$ T. Buchmann, ${ }^{a}$ D. Moseguí González, ${ }^{a}$ L. Song, ${ }^{a}$ Y. Yao, ${ }^{a}$ \\ W. Wang, ${ }^{a}$ J. Werhahn, ${ }^{c}$ G. Santoro, ${ }^{d}$ S. V. Roth, ${ }^{d} H$. Iglev, ${ }^{c}$ R. Kienberger ${ }^{c}$ and \\ P. Müller-Buschbaum*a
}

\begin{abstract}
Titania nanoparticles are produced by laser ablation in liquid in order to initiate functionalization of titania with the polymer for the active layer. By combining these titania nanoparticles and water-soluble poly[3-(potassium-6-hexanoate)thiophene-2,5-diyl] (P3P6T) hybrid solar cells are realized.
\end{abstract}

Due to the ongoing progress in hybrid solar cells, the reached efficiency values become more and more competitive with conventional photovoltaics. ${ }^{1,2}$ The morphology of the active layer turned out to be very important for these devices. ${ }^{3,4}$ Mainly titania $\left(\mathrm{TiO}_{2}\right)$ of different morphologies is used as a major component in such hybrid solar cells. ${ }^{5}$ In addition to research focusing on increasing device efficiencies, the development of alternative, more environmentally friendly hybrid solar cell fabrication routes is interesting. In order to achieve an all-embracing green technology, the materials applied and the required energy for device fabrication are also of interest. Materials in terms of functional components or as additives in the processing should be non-toxic and environmentally friendly. In an optimum approach no organic solvents should be used for the coating of any of the layers of the device. High temperature processing steps should be reduced or avoided to increase the energy payback times of the solar cells. ${ }^{6}$ Following this idea, we present here hybrid solar cells with an active layer based on low temperature processed titania and a watersoluble polymer. The used low temperature route reduces the need of (high temperature) calcination steps in the titania synthesis. In order to achieve bulk heterojunction (BHJ) morphology of the active layer, we apply the method of laser

\footnotetext{
${ }^{a}$ Technische Universität München, Physik-Department, Lehrstuhl für Funktionelle Materialien, James-Franck-Str. 1, 85748 Garching, Germany. E-mail: muellerb@ph.tum.de ${ }^{b}$ Technische Universität München, Munich School of Engineering, Herzig Group, Lichtenbergstr. 4, 85748 Garching, Germany

${ }^{c}$ Technische Universität München, Physik-Department, Lehrstuhl für Laser- und Röntgenphysik, James-Franck-Str. 1, 85748 Garching, Germany

${ }^{d}$ Deutsches Elektronen-Synchrotron (DESY), Notkestr. 85, 22607 Hamburg, Germany $\dagger$ Electronic supplementary information (ESI) available: Full scheme of the production of solar cells, additional spectra and details of the measurement techniques. See DOI: 10.1039/c4nr06782g
}

ablation in liquid to prepare titania nanoparticles. The laser ablation process delivers titania nanoparticles, which are in several aspects suitable for the active layer in hybrid solar cells. One aspect is the size range, which is in the range of the exciton diffusion length within the layer. Another aspect is the electronic properties defined by the crystallinity of the titania. Furthermore, functionalization of the titania nanoparticles with the respective polymer is expected to be beneficial. Thiophene based polymers are of special interest as donor materials. Processability of polythiophenes can be improved by interfacial polymerization ${ }^{7,8}$ or by the introduction of an alkyl group leading to poly(3-alkylthiophene-2,5-diyl) soluble in organic solvents. ${ }^{9}$ For our work of particular interest are water soluble thiophene salts which can be obtained from an oxazoline polythiophene precursor. ${ }^{10}$ Water-soluble polythiophenes would facilitate the achievement of an active layer in the BHJ structure, where the laser-ablated titania nanoparticles are dispersed in a matrix of a hole-conducting polymer. The polymer used as a donor material is poly[3-(potassium-6-hexanoate) thiophene-2,5-diyl] (P3P6T) (Rieke Metals, Inc.) having an average molecular weight $M_{\mathrm{w}}$ of $55-65 \mathrm{~kg} \mathrm{~mol}^{-1}$ and a regioregularity of $82-90 \%$. Together with a gold contact as the top electrode and fluorine-doped tin oxide (FTO) as the bottom electrode the material combination is promising for solar cell application concerning the energy levels. The energy difference between the lowest unoccupied molecular orbital (LUMO) of the donor (P3P6T) and the conduction band of the acceptor $\left(\mathrm{TiO}_{2}\right)$ is more than the needed $0.3 \mathrm{eV}$ for exciton dissociation. ${ }^{11}$ In general, the laser ablation process in liquid produces positively charged particles out of the plasma plume. Therefore, reported laser ablation nanoparticle processes have been successfully expanded by the inclusion of additives to the liquid. As a result ligands are added to the nanoparticles in the process. ${ }^{12}$ The introduction of ligand-free laser-ablated metallic nanoparticles of $\mathrm{Au}, \mathrm{Ag}$ and $\mathrm{Al}$ in organic photovoltaics has been shown to be beneficial for the efficiency of devices. ${ }^{13-15}$ The two different approaches we used for producing laser-ablated titania nanoparticles are summarized in Fig. 1. 

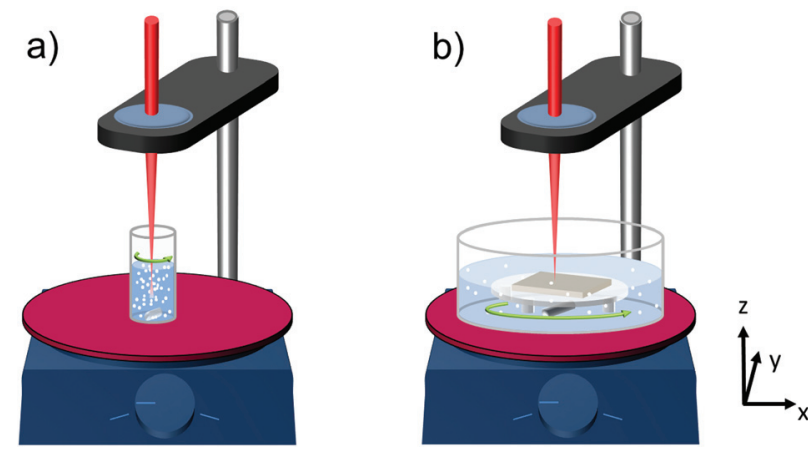

Fig. 1 Basic set-up of the laser ablation process for (a) aqueous titania powder suspension and (b) solid titanium target in water.

On the one hand the processing of titania nanoparticles is carried out starting with a solid target (titanium foil, $0.89 \mathrm{~mm}$ thick, Alfa Aesar), whereas on the other hand titania powder (titanium(Iv) oxide powder in a rutile phase, particle size $<5 \mu \mathrm{m}$, Sigma-Aldrich) in aqueous suspension is the source material for the laser ablation experiment. The laser radiation is provided by using a home-built titanium:sapphire laser system delivering pulses at a central wavelength of $810 \mathrm{~nm}$ with a pulse energy of $290 \pm 20 \mu \mathrm{J}$ and a repetition rate of $1 \mathrm{kHz}$. The pulse duration $t_{\text {pulse }}$ of $270 \pm 50 \mathrm{fs}$ was measured by frequency-resolved optical gating. ${ }^{16}$ The laser beam was focused on the sample via a fused silica lens $(f=100 \mathrm{~mm})$ as shown in Fig. 1. The beam diameter in the focal plane $d_{\text {laser }}=$ $30.9 \pm 0.2 \mu \mathrm{m}$ was determined by using a beam camera (BeamPro, Photon Inc.). The estimated pulse intensity in the focus of about $1.4 \times 10^{13} \mathrm{~W} \mathrm{~cm}^{-2}$ is much higher than the expected ablation threshold. For processing both targets (the titanium dioxide powder dispersed in water and the solid titanium foil, respectively) a stirring plate is applied, as the laser ablation process in liquid flow is more efficient and yields more reproducible results. ${ }^{17}$ In the case of the ablation process from powder (Fig. 1a), a glass vial containing a magnetic stir bar and the titania suspension is placed on the stirring plate. The stirring of the suspension yields the needed flow and the probability of hitting a huge amount of particles with the laser beam is high. Yet not all original powder particles cross the laser focus. For the solid target on the other hand (Fig. 1b), the laser beam would always hit the same point yielding insufficient ablation rates. Thus, the stirring plate with the target holder including the target and the magnetic stir bar is placed on a movable plate. Hence, by adjusting the motion parameters of the motor, the target is moved with respect to the laser focus in $x$ - and $y$-direction yielding a large-area treatment in a circular trace (Fig. 1b). After laser ablation processing of the titanium target or the white titanium dioxide powder, respectively, a bluish suspension develops. This change in color can be attributed to rutile titanium dioxide nanoparticles. ${ }^{18-20}$ Furthermore, the presence of $\mathrm{Ti}^{2+}$ and $\mathrm{Ti}^{3+}$ species might influence the color and was previously found in laser ablation products. ${ }^{19,21}$ Thus, the change in color is a first hint of successful laser ablation of the material.
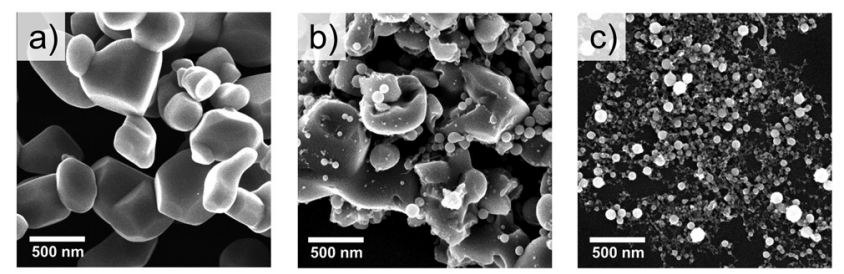

Fig. 2 SEM images of (a) the source titania powder, (b) laser-ablated material from titania powder and (c) laser-ablated material from solid titanium target.

The titanium dioxide powder purchased from Sigma Aldrich is non-spherically shaped, but rather shows rough edges, as depicted in the scanning electron microscopy (SEM) image in Fig. 2a. The size declaration by the manufacturer is $<5 \mu \mathrm{m}$. For the original titania powder a diameter of $(750 \pm 465)$ $\mathrm{nm}$ with very few particles $>3 \mu \mathrm{m}$ is found by SEM analysis. After laser ablation of the material a mean value of $150 \mathrm{~nm}$ with a distinct size distribution is obtained from the SEM images (Fig. 2b). The accumulation of round shaped small particles around larger source particles yields uncertainties for the particle size measurement. In contrast, the particles ablated from a titanium target have a spherical shape with sizes in the range of 5 to $150 \mathrm{~nm}$ with an average diameter of $25 \mathrm{~nm}$ (Fig. 2c).

In order to functionalize the fabricated nanoparticles, the aqueous suspension of the laser-ablated product is removed from the laser beam and an aqueous P3P6T solution is added. A P3P6T:TiO ${ }_{2}$ suspension with a weight ratio of $1: 1$ of the components is achieved. The suspension is put in the laser focus for a short additional treatment in order to enhance the functionalization process. The resulting $\mathrm{P} 3 \mathrm{P} 6 \mathrm{~T}: \mathrm{TiO}_{2}$ compound serves as the active layer in hybrid solar cells. The steps of the solar cell fabrication are shown in Fig. 3. In the chosen inverted cell geometry the electrons are extracted via a transparent conductive oxide (TCO) electrode. As TCO-substrates fluorine doped tin oxide (FTO) is used, since the blocking layer has to be calcined and FTO has better temperature stability as compared with indium tin oxide. A compact titania layer is selected as the hole blocking layer, which reduces the contact of the hole conductor with the FTO and therefore prevents charge recombination (Fig. 3a). The active layer made of the P3P6T:TiO ${ }_{2}$ compound prepared from laser-ablated titania from a titania powder suspension is deposited by spin coating. Thereafter, the sample is transferred to a heating plate at $70^{\circ} \mathrm{C}$ to evaporate the remaining solvent. For the $\mathrm{P} 3 \mathrm{P} 6 \mathrm{~T}: \mathrm{TiO}_{2}$ a)

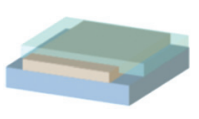

b)

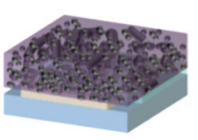

c)

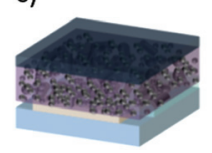

d)

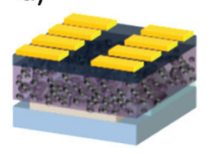

Fig. 3 Built-up of inverted solar cells: (a) the glass substrate with the FTO top layer partially etched away and added compact titania blocking layer; (b) adding the active layer consisting of laser-ablated titania and P3P6T; (c) adding an electron blocking layer; (d) evaporation of gold contacts. 
compound prepared from laser-ablated titania from a solid titanium target, solution casting is chosen due to the lower concentrations achieved in the ablation process (Fig. 3b). An electron blocking layer of poly(3,4-ethylene dioxythiophene): poly(styrenesulfonate) (PEDOT:PSS) is applied from a dilution of PEDOT:PSS with isopropyl alcohol (IPA) in the volume ratio $1: 10$ (Fig. 3c). The last step before characterizing the solar cell is the evaporation of gold top contacts (Fig. 3d) followed by annealing. An annealing step at $140{ }^{\circ} \mathrm{C}$ for $10 \mathrm{~min}$ is chosen as this treatment showed an improvement in crystallinity of the hole-conducting polymer and thus in the performance of solar cells based on poly(3-hexylthiophene) (P3HT), which is similar to the used P3P6T. ${ }^{22}$ All obtained hybrid solar cells are functional. However, the efficiencies $\eta$ achieved for solar cells containing laser-ablated titania out of aqueous titania powder suspension reached only $(0.12 \pm 0.03) \%$. In particular the low short circuit current density $I_{\mathrm{SC}}=(0.36 \pm 0.05) \mathrm{mA} \mathrm{cm}^{-2}$ causes the low efficiency, since open circuit voltage $U_{\mathrm{OC}}=(0.68 \pm 0.03) \mathrm{V}$ and fill factor $\mathrm{FF}=(46 \pm 7) \%$ are both in a very promising range. For hybrid solar cells containing laser-ablated titania from a solid titanium target only a much lower efficiency of $0.02 \%$ could be achieved as a consequence of lower values of $I_{\mathrm{SC}}\left(0.14 \mathrm{~mA} \mathrm{~cm}^{-2}\right), U_{\mathrm{OC}}(0.47 \mathrm{~V})$, and $\mathrm{FF}(29 \%)$.

To understand the reasons which limit the efficiencies of the aqueous processed hybrid solar cells, the crystalline structure of the titania nanoparticles is investigated. X-ray diffraction (XRD) measurements are performed on spin-coated films of P3P6T and the P3P6T:TiO 2 compound based on laser ablation of the titania powder annealed at $120{ }^{\circ} \mathrm{C}$ (Fig. 4a). Additionally, a pure film of laser-ablated $\mathrm{TiO}_{2}$ is cast on a glass substrate and investigated for comparison. The titania film shown in Fig. 4a is not calcined. The theoretical positions for rutile and anatase $\mathrm{TiO}_{2}$ phases ${ }^{23}$ are depicted by green and blue bars, respectively. For the pure titania film, the main crystal phase is rutile. However, a certain amount of anatase phase can be found. For comparison, the material as purchased (without laser ablation) is mainly in its rutile phase. The production of both, the rutile and the anatase phase by laser ablation in solution under controlled conditions was previously observed. ${ }^{24,25}$ After laser ablation of a rutile titania powder target, the presence of rutile, anatase and brookite structure was reported. ${ }^{21}$ The main crystalline phase for titania ablated from a solid target is rutile (see ESI $\dagger$ ). The fabrication of a mixed phase of rutile and anatase by laser ablating a titanium target was observed by Tian et al. ${ }^{26}$ They concluded that the rapidly changing temperature and pressure are responsible for the observed coexistence of both phases. ${ }^{26}$

Although there are several studies on the application of P3P6T in photovoltaics, ${ }^{27-31}$ there are only a few hints in the literature on the crystallinity in the corresponding films, reporting P3P6T to be amorphous. ${ }^{27}$ Accordingly, no literature values for lattice spacings, crystal planes or orientation are available. In our investigation, the pure P3P6T film exhibits (100) and (010) Bragg peaks (Fig. 4a) comparable to those found for P3HT. ${ }^{22}$ For the P3P6T:TiO 2 compound, clear diffraction peaks (indicated by black arrows in Fig. 4a) are observa- a) $\mathrm{q}\left[\hat{\mathrm{A}}^{-1}\right]$
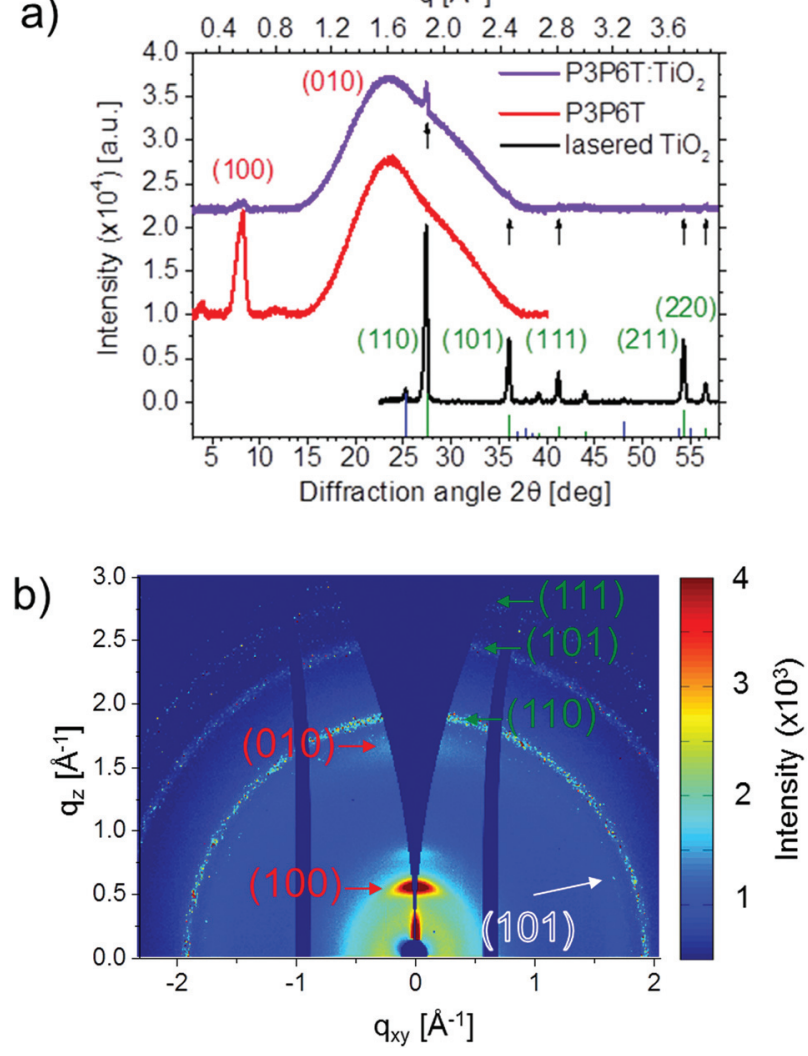

Fig. 4 (a) XRD patterns of laser-ablated $\mathrm{TiO}_{2}$ (black curve), P3P6T (red curve) and the P3P6T:TiO 2 compound (purple curve). Theoretical positions of $\mathrm{TiO}_{2}$ in anatase and rutile phases are plotted in green and blue, respectively. The main rutile peaks, which are observed in the P3P6T:TiO 2 blend are denoted by black arrows. The data are shifted along the $y$-axis for clarity. (b) Two-dimensional GIWAXS pattern of a film of P3P6T:TiO 2 compound. The crystal planes for the polymer are denoted in red letters, whereas the attribution of the observed crystalline phases of $\mathrm{TiO}_{2}$ is noted by green and white letters for the anatase and rutile phase, respectively.

ble, which are caused by $\mathrm{TiO}_{2}$. All observed titania peaks in the P3P6T: $\mathrm{TiO}_{2}$ sample can be attributed to the rutile phase in the (110), (101), (111), (211) and (220) planes. For the pure titania film it is already seen that the rutile phase is dominating, even though the anatase phase is present, too. To further investigate the orientation of the crystals, grazing incidence wide angle X-ray scattering (GIWAXS) measurements are performed using the Micro- and Nanofocus X-ray scattering Instrument $\mathrm{P} 03$ of the PETRA III synchrotron source in Hamburg, Germany. ${ }^{32,33}$ The two-dimensional (2D) GIWAXS data of the P3P6T:TiO 2 film are shown in Fig. $4 \mathrm{~b}$. Due to the powder-like arrangement of the titania particles a ring-shaped intensity distribution of the corresponding Bragg peaks is seen. As for the XRD data, the rutile phases with (101) and (110) Bragg peaks can clearly be identified and are marked in green. Additionally, the (111) Bragg peak of the rutile phase can be identified at the top of the $2 \mathrm{D}$ data. Furthermore, an anatase phase signal, corresponding to the (101) plane, is observed and denoted in blue/white. Higher orders, as seen in 
$\mathrm{XRD}$, are outside the detector range. To quantify the GIWAXS data, cake cuts are performed in vertical $\left(85^{\circ}-95^{\circ}\right)$ and horizontal $\left(1^{\circ}-10^{\circ}\right)$ directions. The lattice spacing for the polymer according to Bragg's equation is $(1.10 \pm 0.01) \mathrm{nm}$ in the [100] direction and $(0.38 \pm 0.01) \mathrm{nm}$ in the [010] direction. Furthermore, the crystallite size is calculated using the Scherrer equation, whereas for a pure P3P6T film the crystallite size for the $(100)$ peak is $(13.9 \pm 0.9) \mathrm{nm}$, it decreases for the sample containing $\mathrm{TiO}_{2}$ to $(7.5 \pm 0.8) \mathrm{nm}$. It is further observed that the intensity of the polymer Bragg peaks decreases by adding $\mathrm{TiO}_{2}$. This indicates less crystallinity of P3P6T due to adding $\mathrm{TiO}_{2}$. Similar observations are found in the literature for P3HT:PCBM blends. ${ }^{34}$ Thus, the diffraction experiments clearly prove the crystallinity of pure P3P6T. In addition, an indication of crystallinity is found from the shoulder of the UV-vis absorption spectra of the pure P3P6T film as well as in the P3P6T:TiO 2 film (Fig. 5a). Furthermore, the titania out of the ablation experiment strongly absorbs below $400 \mathrm{~nm}$, as shown in Fig. 5a. For determining the indirect band gap, the Tauc equation is applied. Averaging over several spectra yields the value of $(2.82 \pm 0.03) \mathrm{eV}$ for the original powder and $(2.5 \pm$ $0.3) \mathrm{eV}$ for the material after laser ablation. Titania in its rutile phase is known to have an indirect band gap of $3.02 \mathrm{eV}$, while in its anatase phase it has an indirect band gap of $3.23 \mathrm{eV}^{35,36}$ For a mixed phase, it is known that a band alignment occurs and a deviation from the pure band gap is possible. ${ }^{26}$ As the rutile phase is thermodynamically more stable, the laserablated particles may be more likely in the rutile than in the anatase phase. Thus, a dominating rutile phase is expected for the laser ablation products as confirmed by X-ray characterization (Fig. 4). However, all the values obtained for the indirect band gap are lower than the expected one for the rutile phase of $3.0 \mathrm{eV}$. Different phases also show higher band gaps. An amorphous phase is expected to have a band gap value of 3.3 $\mathrm{eV}$, and a brookite phase to have a band gap of $3.4 \mathrm{eV} .^{37,38}$ The observed lower value could originate from the lower transmission as the films are turbid and therefore scatter strongly. In the case of the ablated material another factor could play a
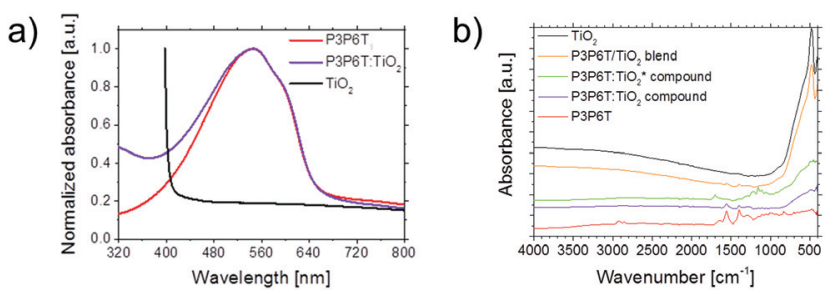

Fig. 5 (a) Absorption spectra normalized to maximum absorbance of laser-ablated $\mathrm{TiO}_{2}$ (black curve), pure P3P6T (red curve), and the P3P6T:TiO 2 compound (purple curve). (b) Infrared spectra of P3P6T and laser-ablated $\mathrm{TiO}_{2}$. The red curve represents pure P3P6T and the black one pure $\mathrm{TiO}_{2}$ by laser ablation from titania powder. A blend of $\mathrm{TiO}_{2}$ laser-ablated from suspension and P3P6T powder (orange), and the corresponding compound of $\mathrm{P} 3 \mathrm{P} 6 \mathrm{~T}: \mathrm{TiO}_{2}$ (purple) are shown. Additionally the compound with $\mathrm{TiO}_{2}$ ablated from a titanium target, denoted $\mathrm{P} 3 \mathrm{P} 6 \mathrm{~T}: \mathrm{TiO}_{2}{ }^{*}$ (green) is shown. Data are shifted along the $y$-axis for clarity. role. As the particles are smaller, more oxygen vacancies might be found. It was shown that the ablation products often contain $\mathrm{Ti}^{3+} \cdot{ }^{21}$ These oxygen vacancy states are located 0.75-1.18 eV below the conduction band. ${ }^{31,39}$ The functionalization of the laser-ablated titanium dioxide particles after mixing with P3P6T and additional laser treatment is verified by Fourier transform infrared (FTIR) spectroscopy. The samples could not be measured as a thin film but on the dry powder extracted from the respective films. To see the effect of functionalization, pristine $\mathrm{P} 3 \mathrm{P} 6 \mathrm{~T}$, pure laser-ablated $\mathrm{TiO}_{2}$ produced out of the titania powder and the corresponding P3P6T: $\mathrm{TiO}_{2}$ compound are investigated. Furthermore, to observe the influence of the subsequent adding and functionalizing of the particles with polymer, pristine P3P6T is mixed with dried laser-ablated $\mathrm{TiO}_{2}$ powder. This sample is denoted as "P3P6T/ $\mathrm{TiO}_{2}$ blend" as it constitutes a simple mixture of the dry components without expecting any special binding between the components. The obtained infrared spectra for the samples using $\mathrm{TiO}_{2}$ powder as ablation target are depicted in Fig. 5b (for zoom in and attributed bands see ESI $\dagger$ ). For $\mathrm{TiO}_{2}$, the signal is obtained at low wavenumbers of $600-400 \mathrm{~cm}^{-1}$. For the polymer, the two ranges $3100-2700 \mathrm{~cm}^{-1}$ for the methylene group, and 1700-1200 $\mathrm{cm}^{-1}$ for the carboxylate group (Fig. 5b) are of interest. Compared with the $\mathrm{P} 3 \mathrm{P} 6 \mathrm{~T}: \mathrm{TiO}_{2}$ compound, no shift or additional absorbance is obtained for the P3P6T: $\mathrm{TiO}_{2}$ blend. However, the signal of $\mathrm{TiO}_{2}$ is strongly suppressed for the P3P6T:TiO 2 compound. This, however, is an indication of the coating of the titania particles but not necessarily of a functionalization, as no absorbance peak is found upon combining the materials. For the compound denoted P3P6T:TiO ${ }_{2}^{*}$ in Fig. 5b prepared with titania nanoparticles ablated from a titanium target, the characteristic carboxyl vibrations of P3P6T at 1395 and $1555 \mathrm{~cm}^{-1}$ are hardly recognizable. However, the polymer is still present in the sample and a new vibration band is observed at $1700 \mathrm{~cm}^{-1}$, which does not exist for the other spectra. Thus, the new vibration band at $1700 \mathrm{~cm}^{-1}$ might be attributed to the coupling of the polymer to the laser-ablated $\mathrm{TiO}_{2}$. This indicates a success in functionalization and coupling of the polymer end-group to the $\mathrm{TiO}_{2}$ nanoparticles. For the previously described sample produced with $\mathrm{TiO}_{2}$ powder as target, it might not be seen, since the ratio of the laser-ablated $\mathrm{TiO}_{2}$ to the original powder after the ablation process is too low for allowing the full amount of the polymer to couple with the ablated $\mathrm{TiO}_{2}$.

\section{Conclusions}

Hybrid solar cells are produced by applying a new water-based processing method, which reduces the need for high temperature steps. Thus, the suggested approach is more environmentally friendly in processing, which will be desired for hybrid solar cell applications. Production of titania nanoparticles via laser ablation with two approaches is tested, using titanium dioxide powder as target and a solid pure titanium target. The key for the active layer is the functionalization of the titania with the polymer P3P6T, which is indicated by FTIR-spec- 
troscopy. The obtained high fill factors and open circuit voltages show the potential of the novel material. Alternative processing of the active layer by spray-coating and printing techniques can improve the applicability of the proposed system.

\section{Acknowledgements}

Financial support by TUM.solar in the frame of the Bavarian Collaborative Research Project "Solar technologies go Hybrid" (SolTec), by the GreenTech Initiative (Interface Science for Photovoltaics - ISPV) of the EuroTech Universities and by the Nanosystems Initiative Munich (NIM) is acknowledged. V.K. thanks the Bavarian State Ministry of Education, Science and the Arts for funding this research work via project "Energy Valley Bavaria”. L.S., W.W. and Y.Y. acknowledge the China Scholarship Council (CSC). The authors thank U. Schwenke and H. Gasteiger for assistance with the FTIR measurements. Portions of this research were carried out at the synchrotron light source PETRA III at DESY. DESY is a member of the Helmholtz Association (HGF).

\section{Notes and references}

1 P. Reiss, E. Couderc, J. De Girolama and A. Pron, Nanoscale, 2011, 3, 446.

2 X. Fan, M. Zhang, X. Wang, F. Yang and X. Meng, J. Mater. Chem. A, 2013, 1, 8694.

3 W. Chen, M. P. Nikiforov and S. B. Darling, Energy Environ. Sci., 2012, 5, 8045.

4 P. Müller-Buschbaum, Adv. Mater., 2014, 26, 7692.

5 M. Rawolle, M. A. Niedermeier, G. Kaune, J. Perlich, M. Memesa, Y.-J. Cheng, J. S. Gutmann and P. MüllerBuschbaum, Chem. Soc. Rev., 2012, 15, 5131.

6 S. B. Darling and F. You, RSC Adv., 2013, 3, 17633.

7 X. G. Li, J. Li and M. R. Huang, Chem. - Eur. J., 2009, 15, 6446.

8 X. G. Li, J. Li, Q.-K. Meng and M.-R. Huang, J. Phys. Chem. $B, 2009,113,9718$.

9 T.-A. Chen, X. Wu and R. D. Rieke, J. Am. Chem. Soc., 1995, 117, 233.

10 R. D. McCullough, P. C. Ewbank and R. S. Loewe, J. Am. Chem. Soc., 1997, 119, 633.

11 M. C. Scharber, D. Mühlbacher, M. Koppe, P. Denk, C. Waldauf, A. J. Heeger and C. J. Brabec, Adv. Mater., 2006, 18, 789.

12 H. Zeng, X.-W. Du, S. C. Singh, S. A. Kulinich, S. Yang, J. He and W. Cai, Adv. Funct. Mater., 2012, 22, 1333.

13 B. Paci, G. D. Spyropoulos, A. Generosi, D. Bailo, V. Rossi Albertini, E. Stratakis and E. Kymakis, Adv. Funct. Mater., 2011, 21, 3573.

14 B. Paci, A. Generosi, V. Rossi Albertini, G. D. Spyropoulos, E. Stratakis and E. Kymakis, Nanoscale, 2012, 4, 7452.

15 G. Kakavelakis, E. Stratakis and E. Kymakis, RSC Adv., 2013, 3, 16288.
16 R. Trebino, K. W. DeLong, D. N. Fittinghoff, J. N. Sweetser, M. A. Krumbügel, B. A. Richman and D. J. Kane, Rev. Sci. Instrum., 1997, 68, 3277.

17 S. Barcikowski, A. Menéndez-Manjón, B. Chichkov, M. Brikas and G. Račiukaitis, Appl. Phys. Lett., 2007, 91, 083113.

18 J. Yao, J. Shao, H. He and Z. Fan, Vacuum, 2007, 81, 1023.

19 P. Liu, W. Cai, M. Fang, Z. Li, H. Zeng, J. Hu, X. Luo and W. Jing, Nanotechnology, 2009, 20, 285707.

20 C.-N. Huang, J.-S. Bow, Y. Zheng, S.-Y. Chen, N. J. Ho and P. Shen, Nanoscale Res. Lett., 2010, 5, 972.

21 E.-C. Chang, B.-C. Lin, P. Shen and S.-Y. Chen, J. Nanosci. Nanotechnol., 2012, 12, 8337.

22 M. A. Ruderer, S. Guo, R. Meier, H.-Y. Chiang, V. Körstgens, J. Wiedersich, J. Perlich, S. V. Roth and P. Müller-Buschbaum, Adv. Funct. Mater., 2011, 21, 3382.

23 ICDD, PCPDFWIN. JCPDS - International Centre for Diffraction Data, 1997.

24 A. Nath, S. S. Laha and A. Khare, Appl. Surf. Sci., 2011, 57, 3118.

25 S. M. Hong, S. Lee, H. J. Jung, Y. Yu, J. H. Shin, K.-Y. Kwon and M. Y. Choi, Bull. Korean Chem. Soc., 2013, 34, 279.

26 F. Tian, J. Sun, J. Yang, P. Wu, H.-L. Wang and X.-W. Du, Mater. Lett., 2009, 63, 2384.

27 G. K. V. V. Thalluri, J.-C. Bolsée, A. Gadisa, M. Parchine, T. Boonen, J. D'Haen, A. E. Boyukbayram, J. Vandenbergh, T. J. Cleij, L. Lutsen, D. Vanderzande and J. Manca, Sol. Energy Mater. Sol. Cells, 2011, 95, 3262.

28 C. D. Danesh, N. S. Starkweather and S. Zhang, J. Phys. Chem. B, 2012, 116, 12887.

29 B. J. Worfolk, D. A. Rider, A. L. Elias, M. Thomas, K. D. Harris and J. M. Buriak, Adv. Funct. Mater., 2011, 21, 1816.

30 L. Baeten, B. Conings, J. D'Haen, A. Hardy, J. V. Manca and M. K. van Bael, Sol. Energy Mater. Sol. Cells, 2012, 107, 230.

31 W. Li, B. J. Worfolk, P. Li, T. C. Hauger, K. D. Harris and J. M. Buriak, J. Mater. Chem., 2012, 22, 11354.

32 A. Buffet, A. Rothkirch, R. Döhrmann, V. Körstgens, M. M. Abul Kashem, J. Perlich, G. Herzog, M. Schwartzkopf, R. Gehrke, P. Müller-Buschbaum and S. V. Roth, J. Synchrotron Radiat., 2012, 19, 647.

33 G. Santoro, A. Buffet, R. Döhrmann, S. Yu, V. Körstgens, P. Müller-Buschbaum, U. Gedde, M. Hedenqvist and S. V. Roth, Rev. Sci. Instrum., 2014, 85, 043901.

34 P. Vanlaeke, A. Swinnen, I. Haeldermans, G. Vanhoyland, T. Aernouts, D. Cheyns, C. Deibel, J. D'Haen, P. Heremans, J. Poortmans and J. Manca, Sol. Energy Mater. Sol. Cells, 2006, 90, 2150.

35 M. Grätzel and F. P. Rotzinger, Chem. Phys. Lett., 1985, 118, 474.

36 D. Reyes-Coronado, G. Rodríguez-Gattorno, M. E. EspinosaPesqueira, C. Cab, R. d. Coss and G. Oskam, Nanotechnology, 2008, 19, 145605.

37 S. Valencia, J. M. Marín and G. Restrepo, Open Mater. Sci. J., 2010, 4, 9.

38 C. Magne, S. Cassaignon, G. Lancel and T. Pauporté, J. Chem. Phys. Phys. Chem., 2011, 12, 2461.

39 D. Cronemeyer, Phys. Rev., 1959, 113, 1222. 